\title{
Game Play Evaluation Metrics
}

\author{
Sagar Gupta \\ B. Tech. CSE Student \\ Department of CSE \& IT \\ Jaypee Institute of Information \\ Technology \\ Noida, UP, India
}

\author{
Prashant Kaushik \\ Assistant Professor \\ Department of CSE \& IT \\ Jaypee Institute of Information \\ Technology \\ Noida, UP, India
}

\author{
Suma Dawn \\ Assistant Professor \\ Department of CSE \& IT \\ Jaypee Institute of Information \\ Technology \\ Noida, UP, India
}

\begin{abstract}
This paper discusses various metrics for game play evaluation based on user categories and market expectation. The use of various metrics improves the game testing ahead of the regular testing methods and also pinpoints the paradigm shift in the user taste. The use of metrics improves game play in conjunction with the user's psychological pattern that is usually age independent. We have also done two case studies which prove the point that the psychological pattern is more important than the age analysis. We have also tried to refine the psychological pattern and predict the next version of game play for the same game. We have made four games using the metrics drafted in this paper and these games are also available on Google Play Store for any reference. In one of the case study we have released 5 version of the same game to see if that verifies the metrics by us.
\end{abstract}

\section{KEYWORDS}

Game-play, Games, Psychology, Game Metrics, Player Engagement, User Experience

\section{INTRODUCTION}

Current generation's psychology is great teacher in making the game metrics and improving the game-play [1-12]. We understand digital games differ by the way they require players to perform recursive actions [17], [20] that lead to polysemic performances and readings [11], [19]. Game research has consequently focused much attention on seeking to develop a better understanding of the different experiential components of gameplay, for example, through flow [7], [18], immersion [6], [13-16], player engagement and many more factors. Useroriented game testing is normally carried out at different stages of the production cycle of digital games, which are commonly produced using agile methodologies. Many methods like instaff testing method paid tester method and outsourced testing methods are employed and along that the tester are given the metrics sheets on which they have to evaluate the game. Many research papers discussed various techniques for making the metrics and many of them have made the test game to show the results. We have also taken two case studies for this purpose. The game-player engagement and other such issues of game testing fall under the broad area of HCI. The data collected in this paper is taken from various users of different age groups and have different life style and psychological patterns. We have also used various control input metrics so that the people are different age group can respond in different manner.

In Nutshell the Game metrics may be said as the quantization of the game development - code, game play, etc. Game metrics are interpretable measures of game parts and are usually calculated as a function of some measurable entity be it hit-tomiss ratio, code loops. Metrics can also be variables or features within the game. A game metric is a quantitative measure of one or more attributes of one or more objects that operate in the context of games.
Games, like any business really, are also prone to those same factors. Particularly since behaviourist design returned to prominence (via social games and now gamification) on the promise of metrics, monetization and new markets, a lot of the same sort of thinking has taken hold. Behaviourists tend to be bottom-line thinkers and conservative game designers. They don't care about emergence or unintended dynamics as those things often get in the way of designing. They want their games to be predictable, marketable to the broadest audience possible and fully understandable. So there are cases where marketing takes over the metrics and all the prediction made by the metrics analysis fails and we may need to redo all metrics including the marketing plan also for undertaking such adjustments.

\section{CURRENT STATE OF THE ART}

There are many areas that have contributed to the growth for learning, implementing and experimenting with Game Metrics. One of the best contributors is the Game Developer's Conference. The main objective of the work done till now has been in the knowing of the games success. For that researchers have categories the metrics in broadly 10 areas. These are:
a) Average time of play.
b) Type of platform on which game is played.
c) The visual appearance of the game.
d) Easability in game installation.
e) Funniness of the game.
f) Sound effects of the game.
g) Average Time required for game to clear the levels.
h) Ease of use in the user controls.
i) Maximum score achieved by the friends of the player.

The last point has been recently invented and very well exploited in recent time and almost all the game now a days have social media plug-in attached to their game which can post the user's score in his/her social media profile. Most systems for metric collection implement some degree of automation. A typical workflow might consist of a human analyst who specifies the desired metrics and then an automated system which then records data and generates corresponding reports and graphs, perhaps using a fixed schedule or game scenarios chosen a priori.

Many companies have made their framework which keeps on collecting their game data and that is mostly in house and not available to the general research.

\section{OUR WORK}

In this section we will discuss the metrics developed by us with the help of case studies we have designed two games one which the usual method and then we evaluate that game using the 
various game metrics designed by us and them we kept on improving the game as per the recommendation to us from the game metrics. The first game is named FlappyJ ver1.1 was designed on simple approach and them it is evaluated with the metrics and them it's we keep on improving this game as well as the metrics also. Finally we have made FlappyJ game ver1.8 also after improving the game on all aspects we made a new game and release it the name of this game is FisheeR both the game are available on Google Play Store.

\subsection{CASE STUDY 1}

In this we have a made a FlappyJ game with similar to popular Flappy Bird game.

This game has 2D bird and its obstacles. This game did not had any splash screen or game end screen. The game is available at the following link

https://play.google.com/store/apps/details?id=com.mm.FlappyJ

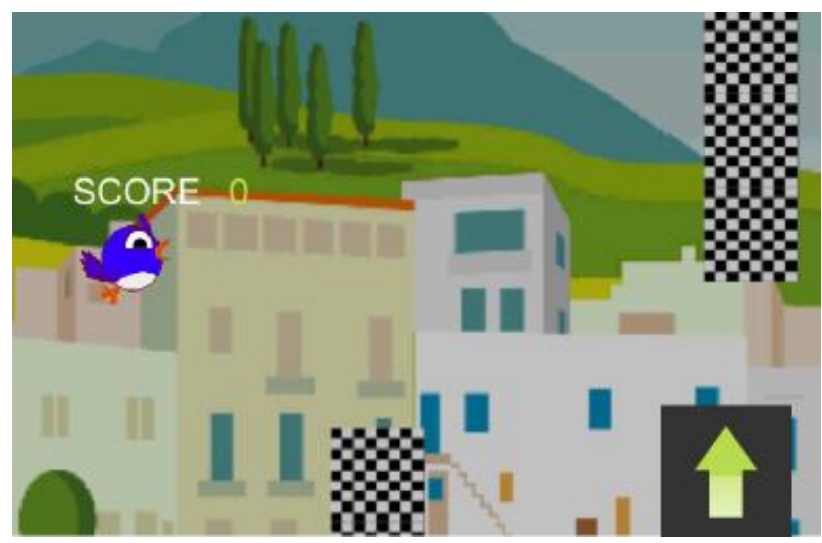

Figure 1: Picture of FlappyJ Ver1.1 With User Control

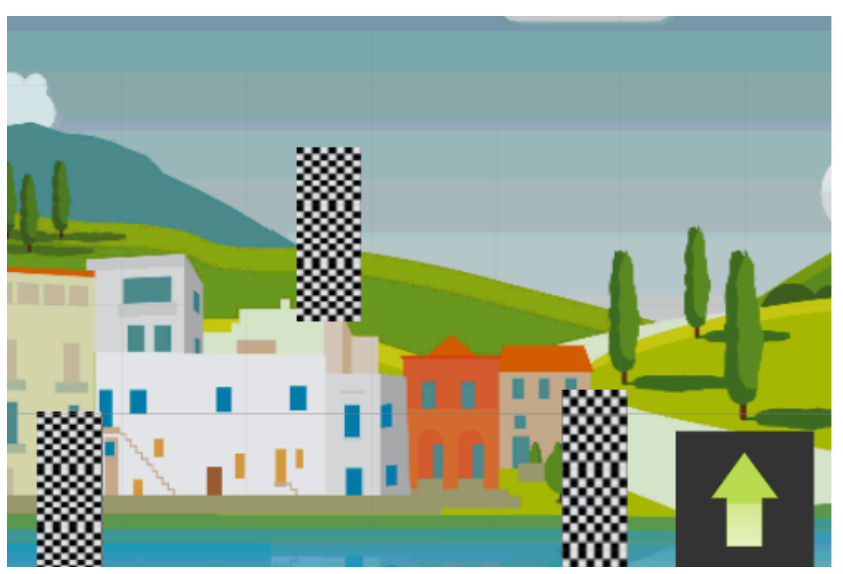

Figure 2: Picture of Environment And User Control (FlappyJ Ver1.1)

Now here are the metrics that are used to evaluate this game and the score which we gets after evaluation of this version is not good so we improve the game and made the new version.

Table 1. User Control Evaluation Metrics

\begin{tabular}{|c|c|}
\hline User Control Type & $\begin{array}{c}\text { Evaluation } \\
\text { Marks }\end{array}$ \\
\hline Two Thumb Control & 1 \\
\hline Two Thumb + 5 Touch & 0.5 \\
\hline Two Thumb + 3 Touch & 1 \\
\hline
\end{tabular}

\begin{tabular}{|c|c|}
\hline Single Touch & 4 \\
\hline Single Touch Anywhere On Screen & 7 \\
\hline Only Sensor Control & 9 \\
\hline
\end{tabular}

Ver1.1 score: 4, Ver. 1.6 score: 7

Table 2. Average Time Before a Player Dies

\begin{tabular}{|c|c|}
\hline Place of Play & Time to die \\
\hline Office & $3 \mathrm{mins}$ \\
\hline Bus/Train & $5 \mathrm{mins}$ \\
\hline Home & $7 \mathrm{mins}$ \\
\hline Before Sleep & $6 \mathrm{mins}$ \\
\hline
\end{tabular}

Ver1.1 score: 2min, Ver. 1.6 score: $7 \mathrm{mins}$

Table 3. Sound Effects Should Be As Per Theme

\begin{tabular}{|c|c|}
\hline $\begin{array}{c}\text { Type Of } \\
\text { Background } \\
\text { Music }\end{array}$ & Evaluation Marks \\
\hline Soothing & 5 \\
\hline Stress Relieving & 6 \\
\hline Joyous & 2 \\
\hline Retarded & 2 \\
\hline Noisy & 5 \\
\hline \multicolumn{2}{|c|}{ Ver1.1 score: 0 (no music), Ver. 1.6 score: 4} \\
\hline
\end{tabular}

Table 4. Social Media Plug-In

\begin{tabular}{|c|c|}
\hline Plug-In Type And Task & $\begin{array}{c}\text { Evaluation } \\
\text { Points }\end{array}$ \\
\hline Login Gives Coin Or Booster & 6 \\
\hline Login Takes Away Posting Rights & -8 \\
\hline Login Post Score & 4 \\
\hline
\end{tabular}

Ver1.1 score: 0 (no plugin), Ver. 1.6 score: 4

Table 5. In Game Advertisement and User's Liking

\begin{tabular}{|c|c|}
\hline Advertisement Position & Evaluation Points \\
\hline During The Game Play & -7 \\
\hline Adv. On Front And Last Screen & 2 \\
\hline Push Notification Advs. & -11 \\
\hline
\end{tabular}

Ver1.1 score: 0 (no adv.), Ver. 1.6 score: 2

\subsubsection{Psychological Feeling of User}

We have assumed that the user is in medium stress before playing the game

Table 6. Psychological Feeling of User

\begin{tabular}{|c|c|c|}
\hline Duration Of Play & $\begin{array}{c}\text { Feeling After The } \\
\text { Game Finishes }\end{array}$ & Points \\
\hline 5mins + Decent Score & Less Stressed & 6 \\
\hline 10mins + Low Score & More Stressed & 2 \\
\hline 15mins+ Decent Score & Less Stressed & 5 \\
\hline
\end{tabular}




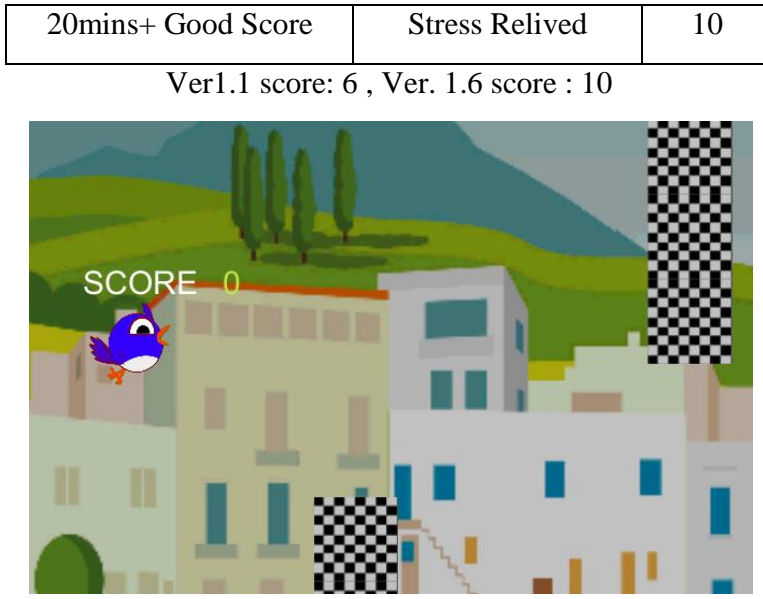

Figure 3: FlappyJ Current Version with New Single Touch Control And Game After Evaluation And Improvement.

This Version Has New User Control And Start And

Finished Screen And Improved Sound And Better Game Play.

Table 7. Score Table Of FlappyJ (Ver. 1.6) Game

\begin{tabular}{|c|c|c|}
\hline Metrics Type & Score & Total \\
\hline User Control & 7 & \\
\hline Average Time & 7 & \\
\hline Sound & 4 & \\
\hline Social Media & 4 & \\
\hline Game Adv. & 2 & \\
\hline Feeling & 10 & 34 \\
\hline Total & & \\
\hline
\end{tabular}

\subsection{CASE STUDY 2}

The snap shot of FisheeR after the improvement of 5 metrics. After learning the results from the first game we have designed the new game with similar theme but new assets and here are the game snap-shots. The FisheeR game is available at the Google play store at the following link:

https://play.google.com/store/apps/details?id=com.mm.FisheeR

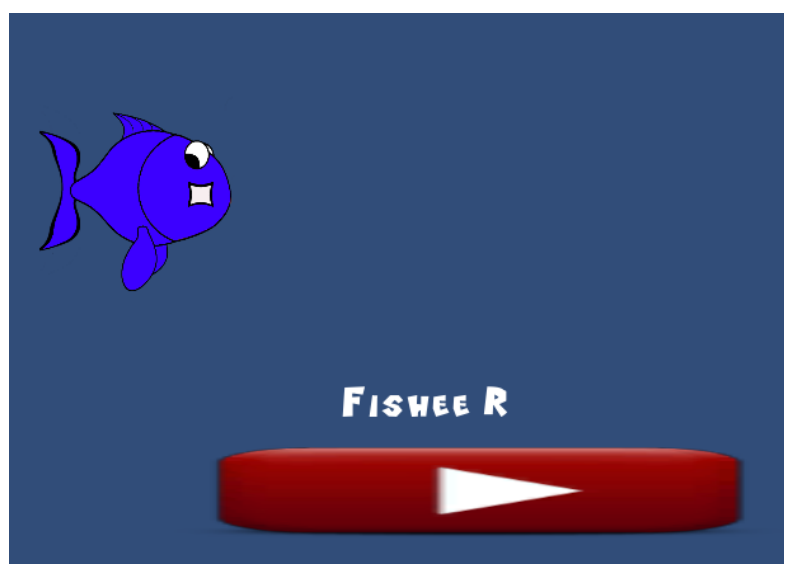

Figure 4: The Splash or Start Screen.

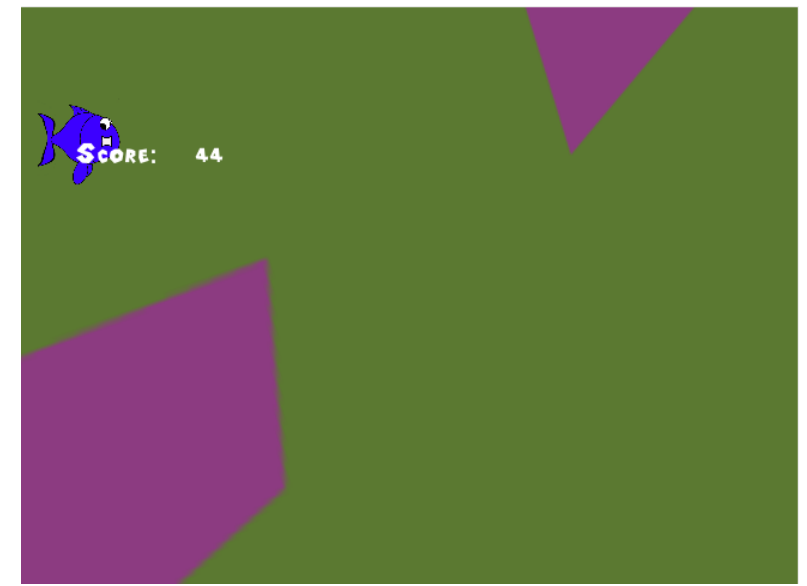

Figure 5: Game-Play Screen

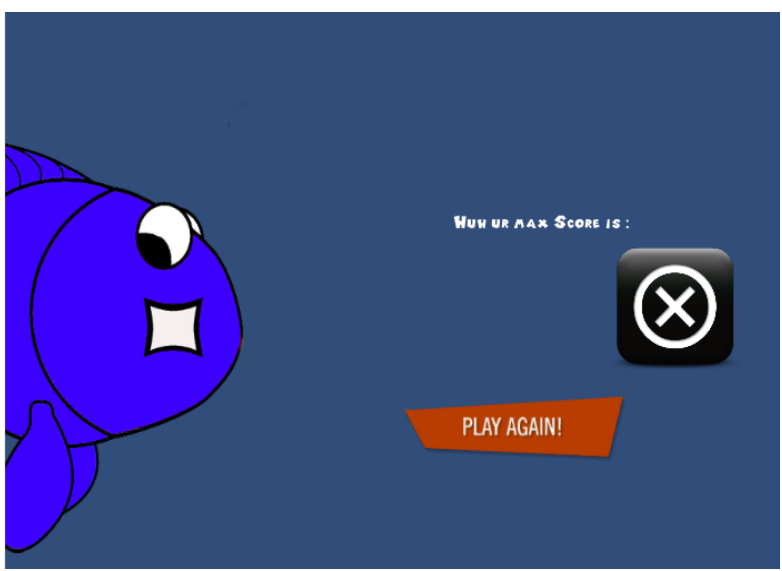

Figure 6: Final /End Screen

Table 8. The Score of the FisheeR Is As Follows

\begin{tabular}{|c|c|c|}
\hline Metrics Type & Score & Total \\
\hline User Control & 8 & \\
\hline Average Time & 7 & \\
\hline Sound & 8 & \\
\hline Social Media & 8 & \\
\hline Game Adv. & 4 & \\
\hline Feeling & 12 & 47 \\
\hline Total & & \\
\hline
\end{tabular}

\subsection{TOOLS USED IN GAME DESIGNING}

We have used Unity 2D game design tool which is new extension to the popular Unity-3d game designing tool and we have also used the Box2D Physics game engine in the game for the better game play.

\section{CONCLUSION}

While concluding the task in this paper we can say that we have designed and tested the updated metrics in the game metrics area which help in finding out the success of the game in the online market and guarantees the good game play of the game. Also we can use these case studies to identify the current psychological pattern in the current age group. Also these metrics helps in identifying the task required to improve the current game in the consideration. 


\section{REFERENCES}

[1] Kim, J. H., Gunn, D. V., Schuh, E., Phillips, B. C., Pagulayan, R. J., Wixon, D. "Tracking Real-Time User Experience (TRUE): A comprehensive instrumentation solution for complex systems". In Proceedings of CHI 2008, Florence, Italy (2008).

[2] Nørgaard, M., Rau, J.: "User Testing in the Combat Zone". In Proceedings of ACE, Toronto, Ontario (2007).

[3] Schultz, C. P.: “Game Testing All in One.” PTR (2005).

[4] Swain, C.: "Master Metrics: The Science behind the Art of Game Design." Presentation at NLGD Conference, Utrecht, Holland (2008).

[5] Thompson, C.: "Halo 3: How Microsoft Labs Invented a New Science of Play." Wired Magazine, Vol. 15, no. 9, (2007)

[6] Tychsen, A., Canossa, A.: "Defining Personas in Games Using Metrics." In: proceedings of Future Play 2008, Toronto, Canada. ACM publishers (2008).

[7] Hoobler, N., Humphreys, G., Agrawala, M.: "Visualizing Competitive Behaviors in Multi-User Virtual Environments." In Proceedings of the IEEE Visualization Conference (2004).

[8] Hurst, A., Hudson, S. E., Mankoff, J.: "Dynamic detection of novice vs. skilled use without a task model." Inproceedings of CHI 2007, ACM Press, pp. 271-280 (2007).

[9] Ferraro P., Robine M., Allali J., Hanna P., and Rocher T.: "Detection of Near-Duplicate Musical Documents from a Multi-Level Comparison of Tonal Information," Information Extraction from the Internet, pp. 129-143, (2011).
[10] Ruch, A.W. "Videogame Interface: Artefacts and Tropes." Videogame Cultures and the Future of Interactive Entertainment Global Conference, Oxford, UK, (2010).

[11] Skalski, P., Whitbred, R.: "Image versus Sound: A Comparison of Formal Feature Effects on Presence and Video Game Enjoyment." PsychNology Journal, (2010).

[12] Waern, A.: "Framing Games", Proceedings of Nordic DiGRA 2012, (2012)

[13] Nacke, L. E., Drachen, A. and Goebel, S. "Methods for Evaluating Gameplay Experience in a Serious Gaming," (2010).

[14] Canossa, A., Drachen, A., Sørensen., J.R.M. (2011)

[15] Arrrgghh!!! - Blending Quantitative and Qualitative.

[16] Methods to Detect Player Frustration, Proc. of FDG 2011, ACM.

[17] Bracken, C.C., Skalski, P.: "Telepresence and Video Games: The Impact of Image Quality." PsychNology Journal, Vol. 7, no. 1, pp. 101-112, (2009).

[18] Aarseth, E.: "I Fought the Law: Transgressive Play and the Implied Player", Proceedings of Digital Game Research Association Conference: Situated Play, (2007).

[19] Ambinder, M.: "Valve's approach to playtesting: The application of empiricism." In Game Developer's Conference, (2009).

[20] Drachen, A., and Canossa, A.: "Analyzing spatial user behavior in computer games using geographic information systems." In Proceedings of the 13th International MindTrek Conference: Everyday Life in the Ubiquitous Era, MindTrek '09, pp. 182-189, (2009). 